\title{
AVALIAÇÃO DO NÍVEL DE ATIVIDADE FÍSICA E FATORES ASSOCIADOS EM ESTUDANTES DE MEDICINA DE FORTALEZA-CE
}

\author{
GRAD. EUTON FREITAS DE CASTRO JÚNIOR \\ Aluno do quinto ano do Curso de Graduação em Medicina da Universidade Estadual do Ceará \\ (UECE) (Fortaleza - Ceará - Brasil) \\ E-mail: castrimjr@hotmail.com
}

GRAD. LEVI ALVES BARRETO

Aluno do quinto ano do Curso de Graduação em Medicina da

Universidade Estadual do Ceará (UECE)

(Fortaleza - Ceará - Brasil)

E-mail: levialvesbarreto@hotmail.com

GRAD. JOSÉ ALBERTO ALVES OLIVEIRA

Médico graduado pela Universidade Estadual do Ceará (UECE)

(Fortaleza - Ceará - Brasil)

E-mail: albertiezzi@hotmail.com

\section{DR. PAULO CÉSAR ALMEIDA}

Doutorado em Saúde Pública pela Universidade de São Paulo (USP). Estatístico.

Docente do Curso de Medicina e do Mestrado de Fisiologia da

Universidade Estadual do Ceará (UECE)

(Fortaleza - Ceará - Brasil)

E-mail: pc49almeida@gmail.com

\section{DR. JOSÉ ALBERTO DIAS LEITE}

Doutorado em Medicina pela Universidade Federal do Rio de Janeiro (UFRJ).

Médico. Especialista em Traumatologia-Ortopedia e em Medicina Física e Reabilitação.

Docente do Curso de Medicina das Universidades Estadual do Ceará (UECE) e Federal do Ceará

(UFC). Chefe do Departamento de Ortopedia da Universidade Federal do Ceará (UFC)

(Fortaleza - Ceará - Brasil)

E-mail: josealberto_leite@hotmail.com

\begin{abstract}
RESUMO
Objetivou-se avaliar o Nível de Atividade Física em acadêmicos de Medicina. Estudo transversal, descritivo e analítico, realizado em escolas médicas públicas e privadas e em hospitaisescola de Fortaleza. Amostra de 840 alunos, 409 do sexo masculino e 431 do feminino, na faixa etária de 17 a 33 anos, escolhidos de forma aleatória. Utilizou-se o Questionário Internacional de Atividade Física na versão curta. A maioria dos estudantes (72,6\%) foi
\end{abstract}


classificada como sedentária ou insuficientemente ativa. Falta de tempo e estética configuram, respectivamente, a principal desmotivação e motivação para a prática de atividade fisica. Necessita-se de mais estudos abordando essa população, com vistas a estabelecer um delineamento mais acurado dos fatores que induzem à inatividade fisica.

PALAVRAS-CHAVE: Atividade fisica; estudantes de medicina; IPAQ; índice de massa corporal.

\section{INTRODUÇÃO}

De acordo com as recomendações atuais evidenciadas no estudo de Haskell et al. (2007), a promoção e a manutenção da saúde em indivíduos adultos saudáveis, de 18 a 65 anos, requisitam atividades físicas aeróbicas de intensidade moderada por, no mínimo, trinta minutos, cinco dias por semana, ou atividade física aeróbica de intensidade vigorosa por, no mínimo, vinte minutos, três dias por semana.

Sabe-se que a prática de atividade física influencia na qualidade de vida dos indivíduos de forma direta, e, ao mesmo tempo, reduz gastos aos cofres públicos e configura-se como um importante artifício da saúde pública. Conforme estudo realizado em 2007, a incorporação no cotidiano da prática de atividade física poderia reduzir a despesa com medicamentos para tratar hipertensão em até $R \$$ | 3.000,00, e no caso de internações por doenças cardiovasculares, a economia seria de $\mathrm{R} \$$ 2. 100.000,00, no total de $\mathrm{R} \$ 4.250 .000,00$ anualmente. Dessa forma, como se depreende, o combate ao sedentarismo na população influencia indiretamente para o progresso econômico. (BIELEMANN; KNUTH; HALLAL, 20 I0).

Entre outros fatores, como afirmam Haskell et al. (2007) e Moretti et al. (2009), a atividade física também é considerada um elemento decisivo na promoção da saúde e na qualidade de vida. No Brasil, as doenças cardiovasculares são as maiores causas de mortes, representando, em 200 I, cerca de $27 \%$ delas. A inatividade física constitui um dos principais fatores de risco relacionados não só às doenças cardiovasculares (MARTINS et al., 20l0), que são doenças crônicas não transmissíveis, mas também a outras doenças enquadradas na mesma categoria, como hipertensão, obesidade, câncer e diabetes. (RIQUE; SOARES; MEIRELLES, 2002). Portanto, a implementação de estratégias que estimulem a atividade física, sobretudo em jovens, é recomendável, conforme pontuado por Enes e Slater (20 I 0).

O estudo de Laporte, Montoye e Caspersen ( 1985) evidenciou mais de trinta técnicas para avaliar o Nível de Atividade Física (NAF), as quais podem variar desde monitorização eletrônica, de custo maior e mais precisa, à aplicação de questionários, menos precisos, entretanto, capazes de avaliar uma maior amostra da população. (MELANSON; FREEDSON, 1996). A escolha do método de mensuração está 
relacionada com o número de indivíduos, o custo e a inclusão de diferentes idades, de acordo com Matsudo et al. (200 I).

Matsudo et al. (200 I) refere, ainda, que o Questionário Internacional de Atividade Física (International Physical Activity Questionnaire - IPAQ) foi desenvolvido para possibilitar o levantamento do NAF em escala mundial, por meio de uma avaliação padronizada. Sua forma curta se configura como uma maneira fácil e rápida de preenchimento, viabilizando estudos de grandes populações e comparações em âmbito internacional.

Embora o IPAQ seja empregado na análise do NAF de alguns grupos (adolescentes e idosos), poucos estudos investigam o NAF na população acadêmica, como observam Silva et al. (2007), especialmente no tocante aos acadêmicos de Medicina.

Consoante evidenciado por pesquisa sobre nível de atividade física de universitários da área de saúde, os do curso médico sobressaem com maior percentual de excesso de peso. (MARCONDELLI; COSTA; SCHMITZ, 2008). Ademais, como mostram Coelho et al. (2005), os estudantes de Medicina apresentam fatores de risco para o desenvolvimento de doenças cardiovasculares, com destaque para o sedentarismo.

Nesse contexto, Huang et al. (2003) salientam que o conhecimento acerca do NAF dessa população é de extrema importância, porquanto esses estudantes estão consolidando seus hábitos de vida, e, assim, ainda é possível um reajuste nos comportamentos sedentários.

O presente estudo tem como objetivo principal avaliar o NAF dos alunos de graduação em Medicina de Fortaleza.

\section{MATERIAIS E MÉTODOS}

\section{DELINEAMENTO DO ESTUDO}

Trata-se de estudo transversal, descritivo e com abordagem quantitativa, desenvolvido em instituições médicas de ensino público e particular, bem como em hospitais-escola, no período de junho de 2007 a março de 2009.

\section{POPULAÇÃO E AMOSTRA}

Devido à inacessibilidade a toda população, uma vez que alguns acadêmicos se encontravam em atividades nas quais não era possível a realização da coleta dos dados, como no auxílio a procedimentos invasivos, os integrantes da amostra foram escolhidos de maneira aleatória simples, utilizando-se para tanto a lista de 
frequência dos universitários. A amostra compôs-se de 840 alunos, sendo 409 do sexo masculino e 431 do sexo feminino, na faixa etária de 17 a 33 anos para ambos os sexos, e média de idade de 21,5 anos ( $D P \pm 2,4$ anos) para homens e 21,4 anos (DP $\pm 2,4$ anos) para mulheres. 58 I eram pertencentes a universidades públicas (395 da Universidade A e 186 da Universidade B) e 259 pertencentes a universidades particulares ( 129 da C e 130 da D), de um total de 1.620, distribuídos do primeiro ao décimo segundo semestre, aos quais se aplicaram os questionários. Como critérios de inclusão adotados, mencionam-se: o aluno estar regularmente matriculado no Curso de Medicina das instituições públicas e privadas de Fortaleza no referido período da pesquisa. Já os critérios de exclusão da amostra foram: estudantes que estavam desenvolvendo as atividades do internato fora de Fortaleza, no período da pesquisa, estudantes que ingressaram na faculdade após o início da coleta e estudantes que se recusaram a participar da pesquisa voluntariamente. $\bigcirc$ significante número de perdas não justificado pelos critérios de exclusão se deve ao fato de que se contou apenas com dois pesquisadores para realizarem a coleta de todos os 1620 alunos.

\section{PROCEDIMENTOS}

Seguindo a resolução 196/96, inerente a pesquisas envolvendo seres humanos, o presente trabalho foi aprovado pelo Comitê de Ética em Pesquisa do Hospital do Câncer/ Instituto do Câncer do Ceará, em reunião de 5 de dezembro de 2007.

Ao final de cada semestre letivo, procedia-se à coleta de dados padronizadamente e autorreferiam-se os valores quantitativos de altura e peso dos indivíduos. Os alunos do primeiro ao quarto ano responderam os questionários nos últimos cinco minutos das aulas, em sala de aula, mediante autorização do professor. No caso dos internos (alunos do quinto e sexto ano), os questionários foram entregues nos respectivos serviços nos hospitais-escola onde eles se encontravam. A fim de se reduzir o número de perdas, questionários incompletos não foram desconsiderados.

As variáveis empregadas para medida do Nível de Atividade Física encontram-se no Questionário Internacional de Atividade Física (IPAQ), o qual foi usado em sua versão curta. Ademais, acrescentaram-se perguntas sobre as características gerais dos estudantes (sexo, idade, peso, altura, faculdade, semestre que cursa, procedência e naturalidade) não utilizadas anteriormente em outro estudo. Na variável sexo, o indivíduo marcava dentre as opções masculino ou feminino. Com relação à idade, relatava, em anos completos, qual possuía no momento da aplicação do questionário, bem como o peso (em quilogramas) e a altura (em metros). Nestas duas últimas, não foi utilizado nenhum meio de mensuração. As variáveis nominais 
faculdade, semestre, procedência e naturalidade foram escritas no questionário pelos estudantes no momento da aplicação do mesmo. Para o cálculo e classificação dos indivíduos quanto ao IMC, adotou-se a equação proposta por Quetelet (http:// www.euro.who.int/en/what-we-do/health-topics/disease-prevention/nutrition/a-healthy-lifestyle/body-mass-index-bmi, acessado em 02 de fevereiro de 2012). Contudo, considerou-se uma mesma categoria de sobrepeso/obesidade, haja vista que a frequência de obesos era muito baixa, inviabilizando as análises estatísticas.

Segundo estabelecido, o IPAQ conceitua e classifica os indivíduos em categorias: Sedentário, Insuficientemente Ativo A, Insuficientemente Ativo B, Ativo e Muito Ativo. (MELANSON; FREEDSON, 1996).

\section{ANÁLISE ESTATIISTICA}

Foram calculadas as seguintes medidas estatísticas: média e desvio padrão das variáveis idade e IMC. Analisou-se a associação entre os níveis de atividade física e as variáveis IMC, período, instituição e sexo por meio do teste de $\chi 2$ (Quiquadrado). As médias de IMC para homens e mulheres foram comparadas pelo teste t de Student para amostras independentes e variâncias desiguais. A normalidade das variáveis idade e IMC não foi testada, haja vista que, pelo Teorema do Limite Central, quando a amostra é muito grande a distribuição tende para a normalidade. (HOEL, 1989). No caso, o tamanho da amostra foi igual a 840. Para todas as análises consideraram-se como estatisticamente significante aquelas com $p<0,05$. Os dados foram processados no SPSS versão 14.0.

\section{RESULTADOS}

Conforme referido, 840 alunos responderam ao questionário. Destes, 409 $(48,7 \%)$ eram do sexo masculino e 431 (51,3\%) do feminino e a idade média da amostra, independente do sexo, foi de 22 anos \pm 2 anos. Já o índice de massa corpórea (IMC) teve média de 24,5 $\pm 3 \mathrm{~kg} / \mathrm{m} 2$ para os homens e $21,2 \pm 2 \mathrm{~kg} /$ $\mathrm{m} 2$ para as mulheres, sendo essa diferença estatisticamente significante $(\mathrm{p}<0$, 05) (Tabela I). 
Tabela I. Média e desvio padrão (DP) das idades do IMC dos estudantes, de acordo com o sexo. Fortaleza, jun.2007/mar.2009

\begin{tabular}{|c|c|c|c|}
\hline \multicolumn{4}{|c|}{ Sexo } \\
\hline Variável & $\begin{array}{c}\text { M } \\
(\text { Média } \pm \text { DP) }\end{array}$ & $\begin{array}{c}F \\
(\text { Média } \pm \text { DP) }\end{array}$ & $P$ \\
\hline Idade & $21,5 \pm 2,4$ & $21,4 \pm 2,4$ & 0,615 \\
\hline IMC & $24,6 \pm 2,7$ & $21,2 \pm 2,1$ & $<0,000$ I* \\
\hline
\end{tabular}

Os resultados de IMC se distribuíram da seguinte forma: baixo peso ( 122 ; 15\%), eutrófico (519; 63,9\%) e sobrepeso/obeso (171; 21,1\%). Ao se fazer a correlação entre a variável IMC com o NAF, observou-se maior concentração na categoria de sedentários (444; 52,7\%), mas não houve diferença estatística significativa entre os grupos ( $p>0,05)$ : baixo peso $(77 ; 63,1 \%)$, eutrófico $(274 ; 52,8 \%)$ e sobrepeso/obeso (93; 54,4\%). (TABELA 2)

Evidencia-se maior proporção de alunos de todos os semestres na categoria de sedentários: início do curso (258; 52,3\%), meio ( I 46; 58,4\%) e final (54; 57,4\%), embora não exista relação estatisticamente significante entre os grupos ( $p>0,05)$. Quando avaliados de acordo com o tipo de instituição, pública ou privada, há maior prevalência de acadêmicos nas categorias sedentário e insuficientemente ativo em ambas: escolas públicas (4I 0; 70,5\%) e escolas privadas (204; 79\%); contudo, os alunos das escolas privadas obtiveram menor NAF quando comparados aos das escolas públicas $(p<0,05)$. Em relação ao gênero, houve maior concentração de discentes do sexo feminino como sedentários $(272 ; 63,1 \%)$ do que do sexo masculino (I 87; 45,7\%). Essa diferença percentual confirmou-se quando foi aplicado o teste de $c 2$, o qual demonstrou ser a amostra feminina menos ativa que a masculina $(p<0,05)$ (TABELA 2). 
Tabela 2. Caracterização dos estudantes quanto ao IMC, semestre letivo, tipo de instituição de ensino (pública ou particular) e gênero em relação ao Nível de Atividade Física. Fortaleza, jun.2007/mar.2009

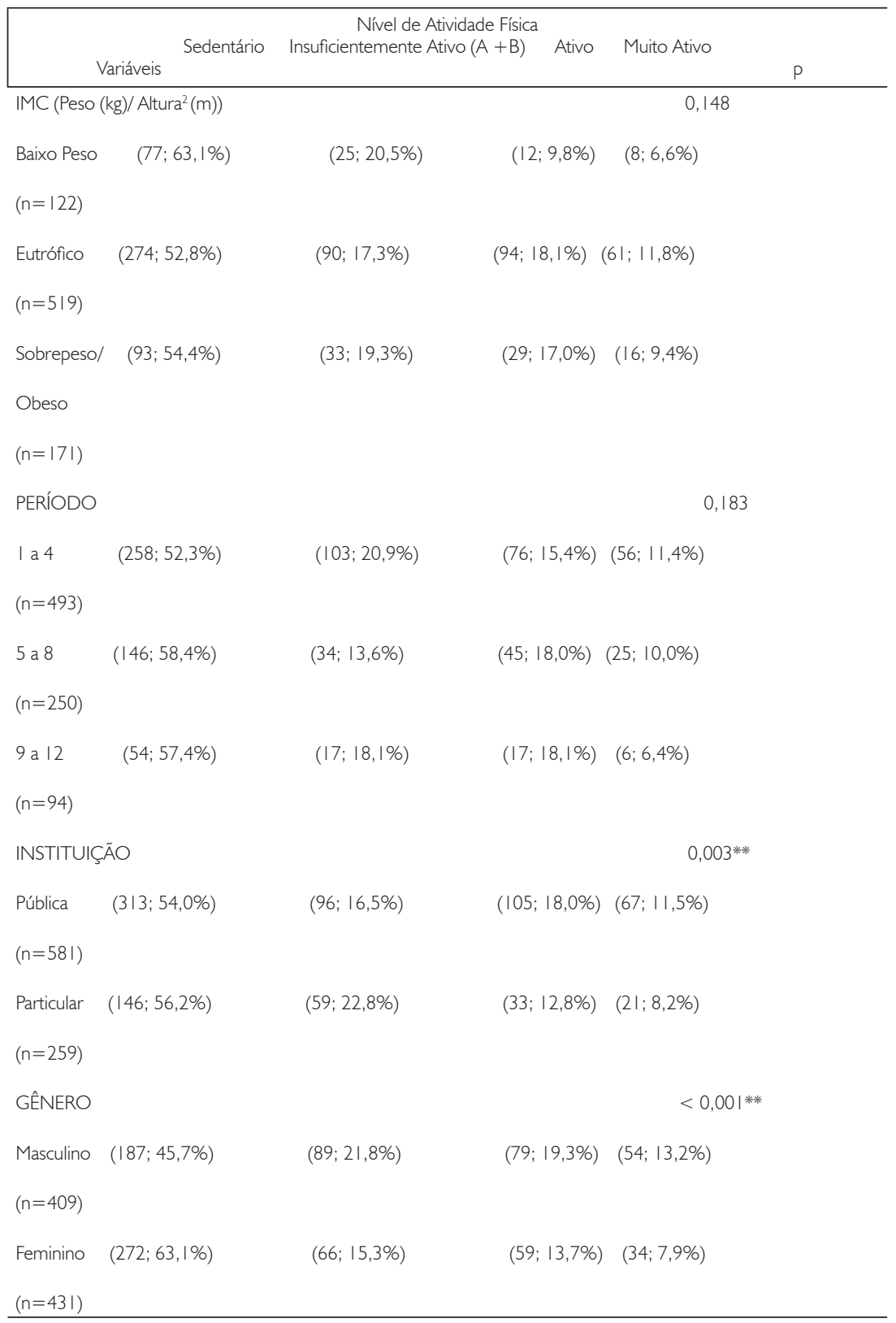

Valor de p pelo teste de $\chi 2$.

IMC = Índice de massa corpórea 
Ao questionar os alunos sobre o que os motiva para a prática de atividades físicas, a maioria respondeu que a procura é por uma melhor condição estética (234; 37,3\%). No entanto, houve uma proporção homogênea entre as três principais respostas: estética corporal, garantir saúde e obter prazer. Quando indagados sobre quais os motivos para a não realização de atividades físicas, a maioria (4I 8; 74,6\%) atribuiu à falta de tempo, pois, segundo os alunos, o Curso de Medicina consome muito tempo das atividades diárias. (TABELA 3).

Tabela 3. Motivos dos estudantes para a prática ou não de atividades físicas. Fortaleza, jun. 2007/mar. 2009****

\begin{tabular}{lcc}
\hline Prá́TICA (Sim - n=627) & $n$ & $\%$ \\
\hline Estética corporal & 234 & 37,3 \\
Garantir saúde & 227 & 36,2 \\
Obter prazer & 157 & 25,0 \\
Outros & 9 & 1,5 \\
\hline Pré́tICA (Não - $\mathrm{n=560)}$ & $\mathrm{n}$ & $\%$ \\
\hline Falta de tempo & 418 & 74,6 \\
Falta de dinheiro & 50 & 8,9 \\
Não gostar & 48 & 8,5 \\
Não achar importante & 4 & 0,8 \\
Outros & 40 & 7,2 \\
\hline \hline
\end{tabular}

*** Os dados dessa tabela incluem mais de uma resposta por aluno, pois, para cada sentença (motivos para a prática ou não de atividade física), o estudante poderia colocar até três itens.

\section{DISCUSSÃO}

A pesquisa avaliou o Nível de Atividade Física dos Estudantes de Medicina de Fortaleza-CE. Fica evidenciado o elevado índice de sedentarismo dessa população (54,6\%), que se revelou ainda maior em discentes de instituições privadas. Os estudos de Fontes e Vianna (2009) e de Marcondelli, Costa e Schmitz (2008) também encontraram alta taxa de sedentarismo entre estudantes da área de saúde, dos quais os de Medicina apresentaram os maiores índices. Ao se observar resultados apresentados por estudantes de Educação Física, consoante se nota, estes são os mais ativos fisicamente, seguidos dos alunos do Curso de Nutrição, na categoria 
ativo, e dos alunos dos Cursos de Enfermagem e Farmácia, na categoria muito ativo. (MARCONDELLI; COSTA; SCHMITZ, 2008). Nesta ótica, supõe-se, os acadêmicos do Curso de Educação Física são mais ativos fisicamente por serem influenciados pelas temáticas especificamente abordadas em sua graduação.

Em corroboração com os estudos de Marcondelli, Costa e Schmitz (2008) e de Salles-Costa et al. (2003), o sedentarismo é maior entre as mulheres (63, I\%). No referente à categoria insuficientemente ativo, no presente estudo, a concentração do sexo feminino é menor ( $15,3 \%$ ) em relação à do sexo oposto, resultado dissonante do trabalho de Silva et al. (2007). Entretanto, pesquisa com I.503 estudantes da Universidade Federal da Paraíba mostrou uma não associação entre o sexo e o baixo Nível de Atividade Física (FONTES; VIANNA, 2009), achado também evidenciado especificamente em estudantes de Educação Física por Silva et al. (2007).

Quanto ao IMC, de acordo com esta pesquisa, 21\% dos estudantes foram classificados como sobrepeso/obeso. Percentual superior ao observado foi encontrado no estudo de Marcondelli, Costa e Schmitz (2008), no qual, entre os cursos da área da saúde, o de Medicina é um dos que apresentam maiores percentuais de excesso de peso (14,6\%); perde apenas para o Curso de Enfermagem (20,4\%). Segundo o mesmo estudo, o Curso de Nutrição possui o maior percentual de estudantes eutróficos e o menor percentual de sobrepeso.

Seo et al. (2007) encontraram uma proporção de $24 \%$ dos estudantes universitários com sobrepeso ou com obesidade. Aqueles com sobrepeso eram mais propensos à inatividade física completa (46\%) do que os participantes de peso normal (37\%). No mesmo estudo, a inatividade física completa durante a graduação foi associada com sobrepeso e com obesidade. Esses dados corroboram em parte com esta pesquisa, pois a proporção de alunos enquadrados nessas categorias foi de $21 \%$, porém a proporção entre discentes sedentários com peso normal $(52,8 \%)$ e com sobrepeso ou obesidade (54,4\%) foi muito próxima. Entretanto, estudo realizado por Conte et al. (2008), em 290 estudantes de Medicina da Universidade de Campinas, mostrou não haver relação entre IMC e NAF. Vale ressaltar que o questionário utilizado nesse estudo não foi o IPAQ. Talvez esse fato possa explicar a diferença de achados, além da amostra ter sido menor do que a do presente estudo.

No presente trabalho, como verificado, não há diferença significativa entre os semestres acerca do Nível de Atividade Física. Achado semelhante ocorreu em estudo na Universidade de Brasília, envolvendo os Cursos da Saúde (MARCONDELLI; COSTA; SCHMITZ, 2008), e na Universidade Federal de Juiz de Fora com estudantes de Educação Física, em estudo de Silva et al. (2007). Em contraposição a esses resultados, os estudos de Buckworth e Nigg (2004), de Desai et al. (2008) e de Fontes e Vianna (2009) mostraram haver uma tendência de diminuição do Nível de 
Atividade Física à medida que os semestres avançam: inicialmente, há uma redução de atividade alta para moderada e depois de moderada para baixa.

Segundo identificado pelo estudo de Reichert et al. (2007), após entrevistar 3. 100 indivíduos, a falta de tempo é a barreira que se associa mais fortemente à inatividade física, dado consonante com esta pesquisa. Para Bowles et al. (2002), argumentar falta de tempo pode ser um reflexo da desmotivação pessoal, não sendo verdadeiramente um obstáculo. Inegavelmente, a falta de tempo no contexto dos estudantes de Medicina constitui uma barreira, pois, de modo geral, as atividades curriculares se desenvolvem em tempo integral. Dessa forma, resta pouco tempo livre para atividades físicas.

Outros motivos apontados para justificar o baixo nível de atividade física, no estudo em foco, foram: falta de dinheiro (8,9\%), não gostar de realizar atividades físicas $(8,5 \%)$, não achar importante $(0,8 \%)$, entre outros (7,2\%). Achados semeIhantes foram identificados em outros estudos, sendo citada também a falta de local adequado. (MARCONDELLI; COSTA; SCHMITZ, 2008). Conforme o estudo de Reichert et al. (2007, p. 5I6), há uma maior prevalência do obstáculo "falta de dinheiro", que é o mais frequente (40,3\%), além de outras barreiras, como: cansaço, falta de companhia e "sentir-se velho".

Todavia, como mostrou estudo desenvolvido na Universidade de Brasília, a classificação socioeconômica não influencia no NAF. (MARCONDELLI; COSTA; SCHMITZ, 2008). Ademais, a falta de dinheiro não impede a realização de atividades físicas, pois há as que requerem poucos investimentos financeiros, como as caminhadas. Consoante determinado estudo (FONTES; VIANNA, 2009), as chances de existir um comportamento de baixa atividade física aumentam em classes sociais mais elevadas.

Em relação aos motivos que impulsionam os estudantes à prática de atividades físicas, o principal apontado neste estudo foi a razão estética (37,3 \%), seguida por "garantir saúde" (36,2\%) e "obter prazer" (25\%). Em estudo com I 5.000 estudantes universitários (KILPATRICK; HEBERT; BARTHOLOMEW, 2005), verificou-se que a atividade física está associada, sobretudo, a motivos extrínsecos, como a aparência, as pressões de saúde, a gestão do stress e o reconhecimento social. E ainda: as razões estéticas predominam entre as mulheres, enquanto os motivos prazer e diversão predominam entre os homens. (MARCONDELLI; COSTA; SCHMITZ, 2008).

\section{CONCLUSÕES}

Conforme é possível concluir, há um elevado índice de inatividade física entre os estudantes de Medicina, cuja maioria, 6 I 4 (73, I\%), foi classificada como sedentária ou insuficientemente ativa. 
Tal como em outros estudos, o sexo feminino mostrou-se mais sedentário $(63,1 \%)$ que o masculino (45,7\%), a evidenciar a consistência do achado. Não houve relação entre as variáveis IMC e semestre cursado com o NAF dos estudantes, e os discentes das instituições particulares se revelaram mais inativos fisicamente que os das públicas.

O principal motivo alegado pelos alunos para a não prática de atividade física foi a falta de tempo (74,6\%), em decorrência da elevada carga horária do curso médico. As razões estéticas foram as maiores motivadoras para a prática de atividade física.

O presente estudo visou contribuir com a literatura vigente, por apresentar dados inéditos referentes a variáveis determinantes do Nível de Atividade Física de estudantes de Medicina. No entanto, salienta-se a necessidade de mais estudos abordando essa população, com vistas a estabelecer um delineamento mais acurado dos fatores que induzem à inatividade física.

\section{Evaluation of the level of physical activity and} associated factors in medical students from Fortaleza - CE.

ABSTRACT: The objective was mainly to evaluate the Physical Activity Level in medical students. A cross-sectional, descriptive and analytical questionnaire was held in public and private medical schools and teaching hospitals of Fortaleza. A sample of 840 students, 409 males and 431 females, aged 1 7-33 years, was selected randomly. We used the International Physical Activity Questionnaire in short version. Most students (72.6\%) were classified as sedentary or insufficiently active. Lack of time and body aesthetic, respectively, are the main demotivation and motivation for physical activity. It is suggested additional studies addressed to this population, to establish a more accurate delineation of the factors that lead to physical inactivity. KEYWORDS: Physical activity; medical students; IPAQ; body mass index.

\section{Evaluación del nivel de actividad física y factores} asociados en estudiantes de medicina de Fortaleza - CE.

RESUMEN: El objetivo era principalmente para evaluar el nivel de actividad física en estudiantes de medicina. Un corte transversal, descriptivo y analítico, que se celebró en las escuelas de medicina públicas y privadas y los hospitales de enseñanza de Fortaleza. Muestra de 840 estudiantes, hombres 409 y 431 mujeres, con edades entre 17-33 años, seleccionados al azar. Se utilizó el Cuestionario Internacional de Actividad Física en su versión corta. La mayoría de los estudiantes (72,6\%) fueron clasificados como sedentarios o activos ins uficientemente. Falta de tiempo y estética, respectivamente, la desAmotivación y la motivación principal para la actividad fisica. Es necesario realizar más estudios frente a esta población, con el fin de establecer una delimitación más precisa de los factores que conducen a la inactividad fisica. PALABRAS CLAVE: Actividad fisica; estudiantes de medicina; IPAQ; índice de masa corporal. 


\section{REFERÊNCIAS}

BIELEMANN, R.; KNUTH, A.; HALLAL, P. Atividade física e redução de custos por doenças crônicas ao Sistema Único de Saúde. Revista Brasileira de Atividade Física e Saúde, Pelotas, v. I5, n. I, p. 9-14, 2010.

BOWLES, $\mathrm{H}$. et al. The association between physical activity behavior and commonly reported barriers in a worksite population. Research Quarterly for Exercise and Sport, Reston, v. 73, p. 464-470, 2002.

BUCKWORTH, J.; NIGG, C. Physical activity, exercise, and sedentary behavior in college students. Journal of American College Health, Hanover, v. 53, n. I, p. 28-34, ago. 2004.

COELHO, V. et al. Perfil lipídico e fatores de risco para doenças cardiovasculares em estudantes de medicina. Arquivos Brasileiros de Cardiologia, Rio de Janeiro, v. 85, n. I, p. 57-62, 2005.

CONTE, M. et al. Nível de atividade física como estimador da aptidão física de estudantes universitários: explorando a adoção de questionário através de modelagem linear. Revista Brasileira de Medicina do Esporte, São Paulo, v. I4, n. 4, p. 332-336, 2008.

DESAI, M. et al. Risk factors associated with overweight and obesity in college students. Journal of American College Health, Hanover, p. 109-1|4, 2008.

ENES, C.; SLATER, B. Obesidade na adolescência e seus principais fatores determinantes. Revista Brasileira de Epidemiologia, São Paulo, v. 13, n. I, p. 163-171, 2010.

FONTES, A.; VIANNA, R. Prevalência e fatores associados ao baixo nível de atividade física entre estudantes universitários de uma universidade pública da região Nordeste - Brasil. Revista Brasileira de Epidemiologia, São Paulo, v. I2, n. I, p. 20-29, 2009.

HASKELL, L. et al. Physical activity and public health: updated recommendation for adults from the American College of Sports Medicine and the American Heart Association. Medicine and Science in Sports and Exercise, Hagerstown, v. 39, n. 8, p. I423-1434, 2007.

HOEL, P. Introduction to mathematical statistics. San Francisco: Wiley, 1989.

HUANG, T. et al. Assessing overweight, obesity, diet, and physical activity in college students. Journal of American College Health, Hanover, v. 52, n. 2, p. 83-86, 2003.

KILPATRICK, M.; HEBERT, E.; BARTHOLOMEW, J. College students' motivation for physical activity: differentiating men's and women's motives for sport participation and exercise. Journal of American College Health, Hanover, v. 54, n. 2, p. 87-94, 2005.

LAPORTE R.; MONTOYE, H.; CASPERSEN, C. Assessment of physical activity in epidemiologic research: problems and prospects. Public Health Reports, Rockville, v. I00, n. 2, p. |3|-|46, 1985.

MARCONDELLI P.; COSTA, T.; SCHMITZ, B. Nível de atividade física e hábitos alimentares de universitários do $3^{\circ}$ ao $5^{\circ}$ semestres da área de saúde. Revista de Nutrição, Campinas, v. 21, n. I, p. 39-47, 2008. 
MARTINS, M. et al. Pressão arterial, excesso de peso e nível de atividade física em estudantes de universidade pública. Arquivos Brasileiros de Cardiologia, São Paulo, v. 95, n. 2, p. 192-199, 2010.

MATSUDO, S. et al. Questionário Internacional de Atividade Física (IPAQ): estudo de validade e reprodutibilidade no Brasil. Revista Brasileira de Atividade Física e Saúde, Pelotas, v. 6, n. 2, p. 5-18, 200|.

MELANSON, E.; FREEDSON, P. Physical activity assessment: a review of methods. Critical Reviews in Food Science and Nutrition. London, p. 385-396, 1996.

MORETTI, A. et al. Práticas corporais/atividade física e políticas públicas de promoção da saúde. Saúde e Sociedade, São Paulo, v. I8, n. 2, p. 346-354, 2009.

REICHERT, F. et al. The role of perceived personal barriers to engagement in leisure-time physical activity. American Journal of Public Health, Boston, v. 97, n. 3, p. 5I 5-519, 2007.

RIQUE, A.; SOARES, E.; MEIRELLES, C. Nutrição e exercício na prevenção e controle das doenças cardiovasculares. Revista Brasileira de Medicina do Esporte, São Paulo, v. 8, n. 2, p. 244-254, 2002.

SALLES-COSTA, R. et al. Gênero e prática de atividade física de lazer. Cadernos de Saúde Pública, Rio de Janeiro, v. 19, n. 2, p. 5325-5333, 2003.

SEO, D. et al. Relations between physical activity and behavioral and perceptual correlates among midwestern college students. Journal of American College Health, Hanover, v. 56, n. 2, p. 187-197. 2007.

SILVA, G. et al. Avaliação do nível de atividade física de estudantes de graduação das áreas saúde/biológica. Revista Brasileira de Medicina do Esporte, São Paulo, v. I3, n. I , p. 39-42, 2007. WORLD HEALTH ORGANIZATION (WHO). Disponível em: < http://www.euro.who. int/en/what-we-do/health-topics/disease-prevention/nutrition/a-healthy-lifestyle/body-mass-index-bmi>. Acesso em: 3 fev. 2012.

Recebido: 19 nov. 201 I

Aprovado: 23 mar. 2012

Endereço para correspondência:

Levi Alves Barreto Av. Soriano Albuquerque, I85, apto. 602

Bairro Joaquim Távora

Fortaleza - CE

CEP: $60130-160$ 